\title{
Mercury, Fatty Acids Content and Lipid Quality Indexes in Muscles of Freshwater and Marine Fish on the Polish Market. Risk Assessment of Fish Consumption
}

\author{
Joanna Łuczyńska ${ }^{1, *}$, Beata Paszczyk ${ }^{1}$, Joanna Nowosad ${ }^{2}$ and Marek Jan Łuczyński ${ }^{3}$ \\ 1 Chair of Commodity and Food Analysis, University of Warmia and Mazury in Olsztyn, \\ ul. Plac Cieszyński 1, 10-726 Olsztyn, Poland; paszczyk@uwm.edu.pl \\ 2 Department of Lake and River Fisheries, University of Warmia and Mazury in Olsztyn, \\ 10-726 Olsztyn, Poland; nowosad.joanna@gamil.com \\ 3 Department of Ichthyology, The Stanisław Sakowicz Inland Fisheries Institute in Olsztyn, \\ 10-719 Olsztyn, Poland; marekjl@infish.com.pl \\ * Correspondence: jlucz@uwm.edu.pl
}

Received: 26 July 2017; Accepted: 19 September 2017; Published: 25 September 2017

\begin{abstract}
Mercury content and fatty acids in muscles of Perca fluviatilis L. (European perch), Leuciscus idus L. (ide), Cyprinus carpio L. (European or common carp), Oncorhynchus mykiss Walb. (rainbow trout), Platichthys flesus L. (European flounder). and Clupea harengus L. (bream) from the Polish market were investigated. The total mercury was processed with AAS. The fatty acids were analyzed by gas chromatography. The concentration of mercury in muscles varied from 0.006 to $0.138 \mathrm{mg} / \mathrm{kg}$ and decreased as follows: perch $\approx$ ide $>$ flounder $>$ herring $\approx$ bream $\approx$ rainbow trout $>\operatorname{carp}(p \leq 0.05)$. There were only significant positive correlations between body weight and mercury content in muscle tissue of carp $(r=0.878)$, flounder $(r=0.925)$ and herring $(r=0.982)$ $(p \leq 0.05)$. The atherogenic index (AI), thrombogenicity index (TI) and flesh-lipid quality index (FLQ) were calculated as follows 0.33-0.70 (IA), 0.16-0.31 (IT) and 13.01-33.22 (FLQ). Hypocholesterolemic (OFA) and hypercholesterolemic fatty acids (DFA) in muscles of fish ranged from 18.26 to 23.01 and from 73.91 to 78.46, respectively. In most cases, there were not significant correlations between size (body weight and total length) and fatty acids in the muscles of the examined fish $(p>0.05)$. The Target Hazard Quotient (THQ) values were below 1, which shows that there is no non-carcinogenic health risk to the consumer by consuming the examined fish.
\end{abstract}

Keywords: fish; mercury; fatty acids; quality index; human health; EWI; THQ

\section{Introduction}

Fish are an important sources of biologically valuable proteins, fats, fat-soluble vitamins and n-3 polyunsaturated fatty acids with five and six double bonds in the carbon chain [1]. The results of prospective cohort studies indicate that consuming fish or fish oil containing the n-3 fatty acids eicosapentaenoic acid (EPA) and docosahexaenoic acid (DHA) is associated with decreased cardiovascular death risk, whereas the consumption of vegetable oil-derived n-3 fatty acid $\alpha$-linolenic acid is not as effective [2]. Long-chain fatty acids can be classified into n-3 fatty acids and n-6 polyunsaturated fatty acids which are beneficial to health, because they have anti-inflammatory properties and decrease the risk of cardiovascular disease, cancer, hypotriglyceridemia and increase HDL cholesterol [3-7]. They also (along with nutrients such as carotenoids, vitamins A, D, E, C and zinc, selenium and iron) influence immune system activity [8]. Saturated fatty acids such as lauric (C12:0), myristic (C14:0) and palmitic (C16:0) acid increase total and LDL-cholesterol concentrations $[9,10]$. 
Stearic acid (C18:0) is neutral or hypocholesterolemic [11-13]. Polyunsaturated fatty acids elicit the most potent hypocholesterolemic effects [14].

In contrast, fish are also vulnerable to some chemical pollutants. It is well known that fish are also an important ecological link in the food chain. They serve as food for other fish, wildlife, and humans and they are indicators of water quality and ecosystem health, because they alert people to changes in water quality [15]. The content of heavy metals, including mercury, discovered in some fish makes it difficult to clearly establish the role of fish consumption on a healthy diet [16], particularly since certain elements, such as mercury (present in organisms of lower trophic levels) can be efficiently transferred to higher-level organisms, becoming more concentrated at the top of the food chain [17]. Human exposure to mercury may occur via a variety of pathways, including consumption of fish, occupational and household uses, dental amalgams and mercury-containing vaccines [18].

Mercury usually combines with other elements to form various inorganic (e.g., the mineral cinnabar, a combination of mercury and sulfur) and organic (e.g., methylmercury-MeHg) compounds, although $\mathrm{Hg}$ occasionally also occurs in its elemental, relatively pure form, as a liquid or vapor [18]. Elemental mercury is lipid soluble and can cross the blood-brain barrier, while inorganic mercury compounds are not lipid soluble, rendering them unable to cross the blood-brain barrier [19]. The high exposure to mercury induces changes in the central nervous system, behavioral changes, tremors, headaches, hearing and cognitive loss, dysarthria, incoordination, hallucinations and death, whereas in the cardiovascular system, this metal induces hypertension in humans and animals [20]. The most dangerous form of mercury is methylmercury. The developing human brain is particularly susceptible to injury caused by $\mathrm{MeHg}$, which easily passes the placental barrier [21]. According to these authors methylmercury can have serious adverse effects on the developing nervous system and may promote heart diseases. This form of mercury is bioaccumulated to a high degree in aquatic food chains to attain its highest concentrations in edible tissues in long-lived predatory fish living in both fresh and ocean waters [22].

In fish, the contribution of methylmercury to total mercury generally ranges between $30 \%$ and $100 \%$, depending on species of fish, size, age and diet. The Panel on Contaminants in the Food Chain (CONTAM) Panel used a conservative approach to calculate methylmercury dietary exposure by assuming that $100 \%$ of mercury in fish is in the form of methylmercury [23]. According this CONTAM Panel, in order to ensure that dietary exposure to inorganic mercury was not underestimated, $20 \%$ of total mercury in fish was simultaneously assumed to be inorganic mercury when calculating inorganic mercury dietary exposure. There are fish species that have low in $\mathrm{Hg}$ (i.e., $<0.1 \mathrm{mg} / \mathrm{kg}$ ) and rich sources of n-3 polyunsaturated fatty acids PUFA. In contrast, other fish species are both highly contaminated with $\mathrm{Hg}(\sim 1 \mathrm{mg} / \mathrm{kg}$ and higher) and are not especially rich sources of $\mathrm{n}-3$ PUFA [24]. The maximum residue level (MRL) of $\mathrm{Hg}$ recommended by EU for fish is $0.5 \mathrm{mg} / \mathrm{kg}$ wet weight and $1 \mathrm{mg} / \mathrm{kg}$ in predatory fish such as shark, swordfish, tuna and pike. Domingo [25] said that adequately balancing adequately the risks and benefits of fish consumption is currently a key nutritional/environmental health issue. Essential aspects in balancing the benefits and risks of regular consumption include the choice of the most suitable fish species and their size (both in terms of PUFA and pollutants, as well as the frequency and amount consumed and the way in which it is served $[13,25]$. The Tolerable Weekly Intake (TWI) for inorganic mercury is expressed as mercury is $4 \mu \mathrm{g} / \mathrm{kg}$ body weight, whereas TWI for methylmercury is expressed as mercury is $1.3 \mu \mathrm{g} / \mathrm{kg}$ body weight [23], whereas the Adequate Intake of $250 \mathrm{mg}$ for eicosapentaenoic acid plus docosahexaenoic acid for adults based on cardiovascular considerations [10]. The objective of this study was to determine the mercury content, profile of fatty acids and the lipid quality indexes (atherogenic index (AI), thrombogenicity index (TI) and flesh-lipid quality index (FLQ) in muscles of important freshwater and marine fish species from the Polish market. Additionally, this study sought to evaluate the dependence between biometric parameters (body weight and total length) and mercury and fatty acids content. The health risk assessment with mercury was determined by using THQ. 


\section{Materials and Methods}

\subsection{Sample Preparation}

Perch (Perca fluviatilis L.), ide (Leuciscus idus L.), carp (Cyprinus carpio L.), rainbow trout (Oncorhynchus mykiss Walb.), flounder (Platichthys flesus L.) and herring (Clupea harengus L.) were bought on the Polish market. The samples of fish were collected the same day. The body weight and total length were measured (Table 1). Muscles (without skin) were dissected from the dorsal part and stored until analysis in the refrigerator at $-18^{\circ} \mathrm{C}$. For large bream, perch, ide, carp and rainbow trout, the samples were prepared from muscles taken from one specimens, whereas for flounder and herring, samples were prepared from muscle tissue taken from two fish.

Ethical statement: according to European and Polish Law, the research done on the commercially catch fishes tissue is free to obtain permission on Local Ethical Commision.

\subsection{Analytical Methods}

\subsubsection{Mercury}

Duplicate samples of muscles were weighed into quartz boats ( $270 \mathrm{mg} \pm 0.0001 \mathrm{~g})$ and transferred from an analytical auto-sampler. The total mercury was processed with atomic absorption spectrometry thermal decomposition using a Milestone DMA-80 (with dual-cell) instrument (Milestone, Sorisole, Italy). The samples are first dried at $160{ }^{\circ} \mathrm{C}$ by $60 \mathrm{~s}$ and then underwent decomposition in a furnace in an oxygen atmosphere (temp. $650{ }^{\circ} \mathrm{C}$ by $60 \mathrm{~s}$ ). The time between the end of drying and the beginning of decomposition $\left(650{ }^{\circ} \mathrm{C}\right)$ is $120 \mathrm{~s}$. The absorption wavelength was $253.65 \mathrm{~nm}$ (detection limit - $0.005 \mathrm{ng} \mathrm{Hg}$ ) and detector was a UV enhanced photodiode instrument. The analysis method was tested by measuring the elements in reference material: BCR CRM 422 (muscles of cod Gadus morhua (L.)) with a certified mercury value. The recovery rate percentage was $100.2 \%(n=4)$.

\subsubsection{Fat and Fatty Acids Analysis}

Approximately $1 \mathrm{~g}$ samples $( \pm 0.0001 \mathrm{~g})$ in duplicate were dried to a constant weight at $105{ }^{\circ} \mathrm{C}$ in glass sample tubes with frits and transferred to weighed beakers. The lipids from the fish muscles (without skin) and liver were extracted according to the hot extraction method using an E-816HE automatic extractor. The analysis consisted of three steps (extraction, rinsing, drying). After the extraction was finished, all of the solvent (petroleum ether) was collected in the tank. Fat was dried in beaker at $100{ }^{\circ} \mathrm{C}$ to a constant weight and was then weighed.

The content of fat $(\%)$ was calculated according to pattern: $x=[(b-a) \times 100] / c$, where: $a=$ weight of flask $(\mathrm{g}), \mathrm{b}=$ weight of flask with extracted fat $(\mathrm{g}), \mathrm{c}=$ weight of samples $(\mathrm{g})$.

The lipids were extracted according to the Folch's procedure [26]. The studied material was broken up and mixed. $2 \mathrm{~g}$ of sample was homogenised for $1 \mathrm{~min}$ with $20 \mathrm{~mL}$ of methanol. Next, $40 \mathrm{~mL}$ chloroform was added and the procedure was continued for $2 \mathrm{~min}$. The prepared mixture was filtered to a $250 \mathrm{~mL}$ glass cylinder. The solid residue was re-suspended in $60 \mathrm{~mL}$ chloroform: methanol $(2: 1 \mathrm{v} / \mathrm{v})$ and homogenized again for $3 \mathrm{~min}$. After filtering, the solid was washed once more with $40 \mathrm{~mL}$ chloroform and once with $20 \mathrm{~mL}$ methanol. The combined filtrate was transferred to the same cylinder. $0.88 \%$ sodium chloride in water (determining $1 / 4$ volume of filtrate) was added to the total filtrate and then shaken and left overnight. The upper layer was removed and to the lower layer a water:methanol mixture $(1: 1 v / v)$ was added and the washing procedure was repeated. The remaining layer was trickled by anhydrous sodium sulphate and distilled by means of aggregate for distillation of solvents. The fatty acid methyl esters were prepared from total lipids with the Peisker method with chloroform:methanol:sulphuric acid (100:100:1 v/v) [27].

The fatty acids of methyl esters of each sample were analyzed using a 7890A chromatograph (Agilent Technologies, Waldbroon, Germany) equipped with a flame-ionization detector (FID) under the following conditions: capillary column (dimension $30 \mathrm{~m} \times 0.25 \mu \mathrm{m}$ with a $0.32 \mathrm{~mm}$ internal 
diameter, liquid phase Supelcowax 10 (Supelco, Bellefonte, PA, USA), temperature: flame-ionization detector $-250{ }^{\circ} \mathrm{C}$, injector $-230^{\circ} \mathrm{C}$, column $-195^{\circ} \mathrm{C}$, carrier gas-helium with a flow rate $1.5 \mathrm{~mL} / \mathrm{min}$. Individual fatty acids were identified by comparing the relative retention time peaks to the known Supelco standards.

\subsection{The Llipid Quality Indexes Were Calculated from the Fatty Acids Composition Using the} Following Formulae

\subsubsection{Index of Atherogenicity (AI)}

The AI indicates the relationship between the sum of the main saturated fatty acids and that of the main classes of unsaturated fatty acids, the former being considered proatherogenic (favoring the adhesion of lipids to cells of the immunological and circulatory system), and the latter antiatherogenic (inhibiting the aggregation of plaque and diminishing the levels of esterified fatty acid, cholesterol, and phospholipids, thereby preventing the appearance of micro and macro coronary diseases) [28-30]

$$
(\mathrm{AI})=[\mathrm{C} 12: 0+(4 \times \mathrm{C} 14: 0)+\mathrm{C} 16: 0] /(\mathrm{n}-3 \mathrm{PUFA}+\mathrm{n}-6 \mathrm{PUFA}+\mathrm{MUFA})
$$

PUFA- polyunsaturated fatty acids

MUFA - monounsaturated fatty acids

C12:0-lauric acid, C14:0—myristic, C16:0-palmitic.

\subsubsection{Index of Thrombogenicity (TI)}

The TI shows the tendency to form clots in the blood vessels. This is defined as the relationship between the prothrombogenetic (saturated) and the antithrombogenetic fatty acids (MUFA, n-6 PUFA and n-3PUFA) [28-30]:

$$
\begin{gathered}
(\mathrm{TI})=[\mathrm{C} 14: 0+\mathrm{C} 16: 0+\mathrm{C} 18: 0] /[(0.5 \times \mathrm{C} 18: 1)+(0.5 \times \text { sum of other MUFA })+ \\
(0.5 \times \mathrm{n}-6 \text { PUFA })+(3 \times \mathrm{n}-3 \text { PUFA })+\mathrm{n}-3 \text { PUFA } / \mathrm{n}-6 \text { PUFA })]
\end{gathered}
$$

\subsubsection{Flesh-Lipid Quality (FLQ)}

The FLQ indicates the percentage correlation between the main n-3 PUFA (EPA + DHA) and the total lipids. The higher value of this index is an indicator of the higher quality of the dietary lipid source [31,32]:

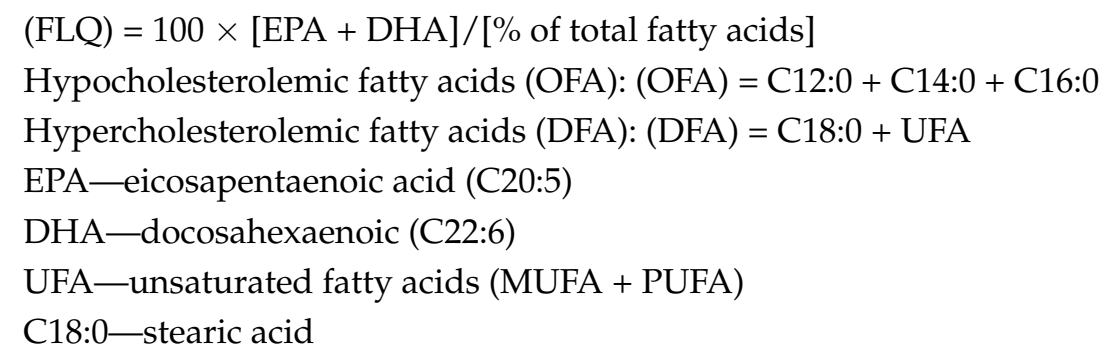

\subsection{Human Health Risk Assessment}

\subsubsection{Estimated Daily Intake of Heavy Metals}

EDI-the estimated daily intake ( $\mu \mathrm{g} / \mathrm{kg}$ body weight/day) $=\mathrm{C} \times \mathrm{IR} / \mathrm{BW}$

TWI-Tolerable Weekly Intake $=$ EDI $\times 7$

$\mathrm{C}$ - the average concentration of heavy metals in food stuffs ( $\mu \mathrm{g} / \mathrm{g}$ wet weight)

IR-the daily ingestion rate ( $\mathrm{g} /$ daily)

The fish consumption was $12.1 \mathrm{~kg}$ per capita/year [33] 
BW—-the average body weight $(60 \mathrm{~kg})$ [34]

\subsubsection{Target Hazard Quotient (THQ)}

The THQ assesses the non-carcinogenic health risk of consumers due to the intake of heavy metal polluted fish using the oral reference dose $\left(\operatorname{RfD}=3.00 \times 10^{-4}\right)[35,36]$. The non-cancer risk model is used in this study because mercury is not classifiable as a human carcinogen. When THQ $<1$ there is health benefit from fish consumption and the consumer is safe, whereas THQ $>1$ suggests a high probability of adverse human health risks:

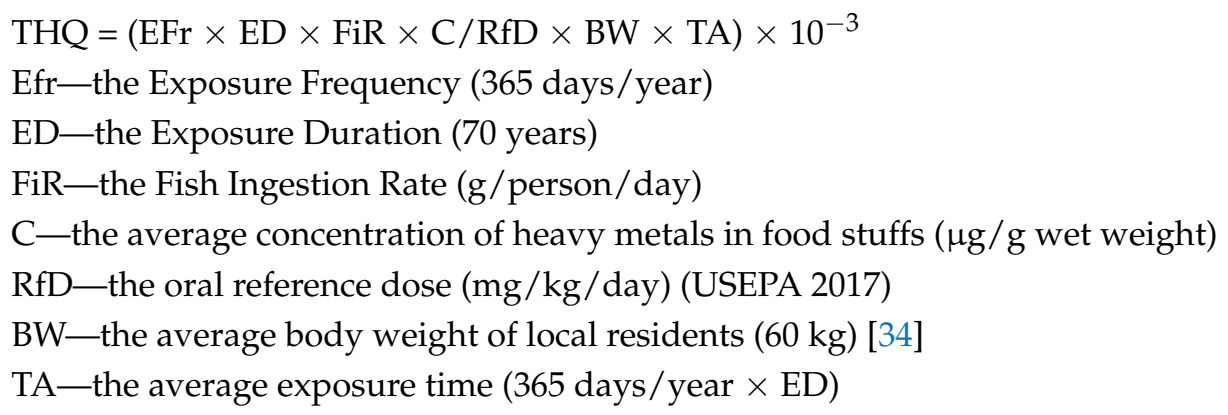

\subsubsection{Statistical Analysis}

Significant interspecific differences in the content of fatty acids and mercury in the muscles were calculated using a one-way analysis of variance ANOVA (Duncan's test) after testing for homogeneity of variance (test Levene's). Differences were found to be significant at $p \leq 0.05$. The correlation coefficients between the content of $\mathrm{Hg}$ and fatty acids in muscles of fish were calculated using the STATISTICA 10 software (StatSoft, Kraków, Poland). Similarly, the correlation coefficients between the content of $\mathrm{Hg}$ and fatty acids in muscle tissue of fish and their size (body weight and total length) were evaluated using the STATISTICA 10 software. The significance level of $p \leq 0.05$ was used.

\section{Results}

The content of total lipid varied widely within and among species (Table 1). Significantly higher values of total lipid were observed in muscles of herring $(p \leq 0.05)$. The content of the element in the muscles of the examined fish was as follows: herring $(11.49 \%)>$ rainbow trout $(2.05 \%) \approx$ flounder $(1.77 \%)$ and flounder $\approx$ ide $(0.80 \%)$ and ide $\approx \operatorname{carp}(0.68 \%) \approx$ perch $(0.35 \%) \approx$ bream $(0.12 \%)$.

Muscles of bream were characterized by a significantly higher content of saturated fatty acids SFA $(32.94 \%)(p \leq 0.05)$, whereas the content of monounsaturated fatty acids MUFA $(54.54 \%)$ was significantly higher in muscle tissue of herring $(p \leq 0.05)$ than in the other fish examined (Table 2$)$. In the case of bream and perch as representative wild freshwater fish, the muscles of these fish contained significantly more n-3 PUFA and n-3 HUFA than marine fish (flounder and herring), cultured fish (carp and rainbow trout) and ide inhabiting different aquatic ecosystems (river and lakes) $(p \leq 0.05)$. The values n-3 PUFA in muscle tissue of bream and perch were $37.46 \%$ and $38.62 \%$, while the contents of n-3 HUFA were $35.00 \%$ and $36.57 \%$, respectively. However, the muscles of bream, carp and rainbow trout had significantly higher content of n-6 PUFA $(15.48 \%, 15.10 \%$ and $15.03 \%$, respectively).

A significantly lower amount of hypocholesterolemic fatty acids was observed in muscles of rainbow trout $(18.26 \%)$ and flounder $(20.34 \%)(p \leq 0.05)$ than other fish examined, although there were no significant differences between muscle tissue flounder and carp, ide, and bream $(p>0.05)$ (Table 2). hypercholesterolemic fatty acids contents in the muscles of fish were as follows: flounder $(78.46 \%)$ $\approx \operatorname{carp}(77.55 \%) \approx$ ide $(77.33 \%)$ and carp $\approx$ ide $\approx$ herring $(76.07 \%) \approx$ perch $(75.73 \%)$ and herring $\approx$ perch $\approx$ bream $(74.88 \%) \approx$ rainbow trout $(73.91 \%)$. The muscles of herring had significantly higher index of atherogenicity $(0.70)$ than other fish studied $(p \leq 0.05)$, whereas the muscle tissue of carp had a significantly higher index of thrombogenicity $(0.31)(p \leq 0.05)$. There were also significant differences between the value of flesh-lipid quality in perch (33.22) and other fish examined ( $p \leq 0.05)$. 
Table 1. Mercury and total lipids content (mean \pm SD, range), and linear correlation coefficients (r) between body weight or total length and content of mercury in muscles of fish.

\begin{tabular}{|c|c|c|c|c|c|c|c|c|c|}
\hline Species & $\begin{array}{c}\text { Body Weight } \\
(\mathrm{g})\end{array}$ & $\begin{array}{l}\text { Total Length } \\
\text { (cm) }\end{array}$ & Total Lipids (\%) & $\begin{array}{c}\mathrm{Hg} \text { (mg/kg Wet } \\
\text { Weight) }\end{array}$ & $\mathrm{Hg}$ (mg/100g Fat) & Body Weight (r) & $p$ & $\begin{array}{l}\text { Total Length } \\
\text { (r) }\end{array}$ & $p$ \\
\hline Bream Abramis brama L. $n=6$ & $\begin{array}{c}207.7 \pm 20.5 \\
192.0-248.0\end{array}$ & $\begin{array}{l}26.7 \pm 1.4 \\
25.5-29.5\end{array}$ & $\begin{array}{c}0.115 \pm 0.050 \mathrm{~d} \\
0.080-0.200\end{array}$ & $\begin{array}{c}0.016 \pm 0.009 c \\
0.006-0.027\end{array}$ & $\begin{array}{c}1.467 \pm 0.904 \\
0.550-3.000\end{array}$ & 0.580 & 0.227 & 0.476 & 0.340 \\
\hline Perch Perca fluviatilis L. $n=5$ & $\begin{array}{l}561.2 \pm 155.1 \\
296.0-704.0\end{array}$ & $\begin{array}{l}32.7 \pm 2.6 \\
28.0-34.5\end{array}$ & $\begin{array}{c}0.352 \pm 0.134 \mathrm{~d} \\
0.230-0.560\end{array}$ & $\begin{array}{c}0.138 \pm 0.111 \mathrm{a} \\
0.078-0.336\end{array}$ & $\begin{array}{l}4.160 \pm 3.262 \\
1.875-9.882 \\
\end{array}$ & 0.601 & 0.283 & 0.459 & 0.437 \\
\hline Ide Leuciscus idus L. $n=6$ & $\begin{array}{l}950.0 \pm 179.3 \\
742.0-1266.0 \\
\end{array}$ & $\begin{array}{l}40.6 \pm 3.1 \\
36.0-43.8\end{array}$ & $\begin{array}{l}0.802 \pm 0.378 \mathrm{~cd} \\
0.400-1.400\end{array}$ & $\begin{array}{c}0.109 \pm 0.050 \mathrm{a} \\
0.046-0.161\end{array}$ & $\begin{array}{c}1.716 \pm 1.283 \\
0.357-4.025\end{array}$ & 0.787 & 0.063 & 0.183 & 0.729 \\
\hline Carp Cyprinus carpio L. $n=5$ & $\begin{array}{l}1197.2 \pm 198.8 \\
938.0-1432.0\end{array}$ & $\begin{array}{l}34.6 \pm 0.5 \\
34.0-35.2\end{array}$ & $\begin{array}{c}0.684 \pm 0.494 \mathrm{~d} \\
0.210-1.470\end{array}$ & $\begin{array}{c}0.006 \pm 0.002 \mathrm{~d} \\
0.004-0.009\end{array}$ & $\begin{array}{c}0.158 \pm 0.161 \\
0.041-0.429\end{array}$ & 0.878 & 0.050 & 0.683 & 0.204 \\
\hline $\begin{array}{c}\text { Rainbow trout Oncorhynchus } \\
\text { mykiss Walb. } n=6\end{array}$ & $\begin{array}{l}202.7 \pm 33.8 \\
158.0-238.0\end{array}$ & $\begin{array}{l}25.3 \pm 1.0 \\
24.0-26.2\end{array}$ & $\begin{array}{c}2.055 \pm 0.368 \mathrm{~b} \\
1.470-2.460\end{array}$ & $\begin{array}{c}0.015 \pm 0.001 \mathrm{c} \\
0.013-0.016\end{array}$ & $\begin{array}{c}0.073 \pm 0.010 \\
0.063-0.088\end{array}$ & 0.429 & 0.396 & 0.336 & 0.514 \\
\hline Flounder Platichthys flesus L. $n=12$ & $\begin{array}{l}274.1 \pm 64.9 \\
195.0-369.0\end{array}$ & $\begin{array}{l}28.6 \pm 1.6 \\
27.0-31.7\end{array}$ & $\begin{array}{c}1.770 \pm 0.719 \mathrm{bc} \\
0.850-2.340\end{array}$ & $\begin{array}{c}0.056 \pm 0.020 \mathrm{~b} \\
0.028-0.084\end{array}$ & $\begin{array}{c}0.354 \pm 0.164 \\
0.221-0.671\end{array}$ & 0.925 & 0.008 & 0.600 & 0.208 \\
\hline Herring Clupea harengus L. $n=12$ & $\begin{array}{l}182.9 \pm 30.2 \\
142.0-227.0\end{array}$ & $\begin{array}{l}26.1 \pm 0.9 \\
24.9-27.6\end{array}$ & $\begin{array}{c}11.487 \pm 1.834 \mathrm{a} \\
9.620-14.560\end{array}$ & $\begin{array}{c}0.021 \pm 0.012 \mathrm{c} \\
0.007-0.039\end{array}$ & $\begin{array}{c}0.018 \pm 0.009 \\
0.006-0.033\end{array}$ & 0.982 & 0.0005 & 0.794 & 0.059 \\
\hline
\end{tabular}

$n-$ Number of fish; SD—standard deviation; $\mathrm{a}, \mathrm{b}, \mathrm{c}, \mathrm{d}$-significant differences at $p \leq 0.05$. The same letter indicates a lack of significant differences between the muscles fish species $(p>0.05) ; p$ - significance levels for the correlation between the content of mercury in muscles of fish and their body weight or total length.

Table 2. Lipid content (\%) and fatty acids composition (\% of total fatty acids) in muscles of different fish species.

\begin{tabular}{|c|c|c|c|c|c|c|c|c|c|c|c|c|c|c|}
\hline \multirow{2}{*}{ Fatty Acids } & \multicolumn{2}{|c|}{ Bream } & \multicolumn{2}{|c|}{ Perch } & \multicolumn{2}{|c|}{ Ide } & \multicolumn{2}{|c|}{ Carp } & \multicolumn{2}{|c|}{ Rainbow Trout } & \multicolumn{2}{|c|}{ Flounder } & \multicolumn{2}{|c|}{ Herring } \\
\hline & $\bar{x}$ & SD & $\bar{x}$ & SD & $\bar{x}$ & SD & $\bar{x}$ & SD & $\bar{x}$ & SD & $\bar{x}$ & SD & $\bar{x}$ & SD \\
\hline$n$ & \multicolumn{2}{|c|}{6} & \multicolumn{2}{|c|}{5} & \multicolumn{2}{|c|}{6} & \multicolumn{2}{|c|}{5} & \multicolumn{2}{|c|}{6} & \multicolumn{2}{|c|}{12} & \multicolumn{2}{|c|}{12} \\
\hline fat & 0.12 & 0.05 & 0.35 & 0.13 & 0.80 & 0.38 & 0.68 & 0.49 & 2.06 & 0.37 & 1.77 & 0.72 & 11.49 & 1.83 \\
\hline $\mathrm{C} 12: 0$ & $0.11 \mathrm{ab}$ & 0.04 & $0.07 \mathrm{~cd}$ & 0.00 & $0.11 \mathrm{ab}$ & 0.04 & $0.10 \mathrm{bc}$ & 0.03 & $0.05 \mathrm{~d}$ & 0.01 & $0.08 \mathrm{bcdd}$ & 0.02 & $0.14 \mathrm{a}$ & 0.02 \\
\hline C14:0 & $0.82 \mathrm{e}$ & 0.17 & $0.97 \mathrm{de}$ & 0.13 & $1.47 \mathrm{cde}$ & 0.60 & $1.58 \mathrm{~cd}$ & 0.39 & $1.93 \mathrm{c}$ & 0.11 & $3.51 \mathrm{~b}$ & 0.26 & $9.77 \mathrm{a}$ & 1.13 \\
\hline C15:0 & $0.91 \mathrm{a}$ & 0.19 & $0.47 \mathrm{c}$ & 0.05 & $0.59 \mathrm{bc}$ & 0.19 & $0.45 c$ & 0.05 & $0.18 \mathrm{~d}$ & 0.01 & $0.72 \mathrm{~b}$ & 0.10 & $0.49 c$ & 0.05 \\
\hline $\mathrm{C} 16: 0$ & $21.27 \mathrm{ab}$ & 1.51 & $21.97 \mathrm{a}$ & 1.80 & $19.75 b$ & 2.29 & $19.39 \mathrm{~b}$ & 1.07 & $16.28 \mathrm{c}$ & 0.61 & $16.74 \mathrm{c}$ & 1.18 & $13.00 \mathrm{~d}$ & 1.54 \\
\hline C17:0 & $1.63 \mathrm{~b}$ & 0.15 & $0.68 \mathrm{c}$ & 0.05 & $0.61 \mathrm{~cd}$ & 0.15 & $0.66 \mathrm{c}$ & 0.11 & $3.43 \mathrm{a}$ & 0.38 & $0.40 \mathrm{de}$ & 0.06 & $0.20 \mathrm{e}$ & 0.03 \\
\hline $\mathrm{C} 18: 0$ & $7.82 \mathrm{a}$ & 0.46 & $5.74 \mathrm{bc}$ & 0.49 & $5.19 \mathrm{c}$ & 1.49 & $6.04 \mathrm{~b}$ & 0.48 & $0.18 \mathrm{f}$ & 0.01 & $2.88 \mathrm{~d}$ & 0.43 & $0.99 \mathrm{e}$ & 0.07 \\
\hline $\mathrm{C} 20: 0$ & $0.17 \mathrm{~cd}$ & 0.04 & $0.11 \mathrm{~d}$ & 0.02 & $0.15 \mathrm{~d}$ & 0.06 & $0.27 \mathrm{bc}$ & 0.04 & $4.06 \mathrm{a}$ & 0.20 & $0.09 \mathrm{~d}$ & 0.02 & $0.33 \mathrm{~b}$ & 0.05 \\
\hline $\mathrm{C} 22: 0$ & $0.20 \mathrm{a}$ & 0.05 & $0.01 \mathrm{c}$ & 0.00 & $<0.01 \mathrm{c}$ & 0.00 & $<0.01 \mathrm{c}$ & 0.00 & $0.16 \mathrm{~b}$ & 0.02 & $<0.01 \mathrm{c}$ & 0.00 & $<0.01 \mathrm{c}$ & 0.00 \\
\hline$\Sigma$ SFA & $32.94 \mathrm{a}$ & 2.18 & $30.01 \mathrm{~b}$ & 2.25 & $27.86 \mathrm{bc}$ & 2.57 & $28.49 \mathrm{bc}$ & 1.14 & $26.27 \mathrm{~cd}$ & 0.67 & $24.42 \mathrm{~d}$ & 1.62 & $24.92 \mathrm{~d}$ & 2.64 \\
\hline C14:1 & $0.01 \mathrm{c}$ & 0.00 & $0.05 \mathrm{~b}$ & 0.02 & $0.06 \mathrm{ab}$ & 0.04 & $0.06 \mathrm{ab}$ & 0.03 & $0.02 \mathrm{c}$ & 0.00 & $0.08 \mathrm{a}$ & 0.02 & $0.08 \mathrm{a}$ & 0.01 \\
\hline C16:1 & $2.84 \mathrm{~d}$ & 0.55 & $5.46 c$ & 2.11 & $7.65 \mathrm{~b}$ & 0.88 & $10.49 \mathrm{a}$ & 1.22 & $0.12 \mathrm{e}$ & 0.01 & $21.17 \mathrm{a}$ & 3.25 & $4.60 \mathrm{~cd}$ & 0.39 \\
\hline
\end{tabular}


Table 2. Cont.

\begin{tabular}{|c|c|c|c|c|c|c|c|c|c|c|c|c|c|c|}
\hline \multirow{2}{*}{ Fatty Acids } & \multicolumn{2}{|c|}{ Bream } & \multicolumn{2}{|c|}{ Perch } & \multicolumn{2}{|c|}{ Ide } & \multicolumn{2}{|c|}{ Carp } & \multicolumn{2}{|c|}{ Rainbow Trout } & \multicolumn{2}{|c|}{ Flounder } & \multicolumn{2}{|c|}{ Herring } \\
\hline & $\bar{x}$ & SD & $\bar{x}$ & SD & $\bar{x}$ & SD & $\bar{x}$ & SD & $\bar{x}$ & $\mathrm{SD}$ & $\bar{x}$ & SD & $\bar{x}$ & SD \\
\hline$n$ & \multicolumn{2}{|c|}{6} & \multicolumn{2}{|c|}{5} & \multicolumn{2}{|c|}{6} & \multicolumn{2}{|c|}{5} & \multicolumn{2}{|c|}{6} & \multicolumn{2}{|c|}{12} & \multicolumn{2}{|c|}{12} \\
\hline C17:1 & $0.80 \mathrm{~cd}$ & 0.13 & $0.61 \mathrm{de}$ & 0.10 & $0.88 \mathrm{bc}$ & 0.32 & $1.09 \mathrm{ab}$ & 0.25 & $0.31 \mathrm{f}$ & 0.01 & $1.17 \mathrm{a}$ & 0.21 & 0.54 ef & 0.09 \\
\hline C18:1 & $9.73 \mathrm{e}$ & 1.06 & $12.20 \mathrm{~d}$ & 3.36 & $17.21 \mathrm{c}$ & 1.03 & $22.74 \mathrm{~b}$ & 2.70 & $27.78 \mathrm{a}$ & 1.72 & $16.35 \mathrm{c}$ & 1.69 & $7.15 \mathrm{f}$ & 0.74 \\
\hline C20:1(n-7) & $0.25 \mathrm{~b}$ & 0.10 & $0.12 \mathrm{~b}$ & 0.01 & $0.26 \mathrm{~b}$ & 0.06 & $0.23 \mathrm{~b}$ & 0.04 & $0.08 \mathrm{~b}$ & 0.01 & $3.45 \mathrm{a}$ & 1.02 & $0.17 \mathrm{~b}$ & 0.02 \\
\hline C20:1(n-9) & $0.18 \mathrm{e}$ & 0.11 & $0.33 \mathrm{de}$ & 0.10 & 0.65 cde & 0.08 & 1.38 & $0.37 \mathrm{~b}$ & $0.84 \mathrm{bcd}$ & 0.09 & $1.05 \mathrm{bc}$ & 0.25 & $14.99 \mathrm{a}$ & 1.03 \\
\hline $\mathrm{C} 20: 1(\mathrm{n}-11)$ & $0.33 c$ & 0.12 & $<0.01 \mathrm{~d}$ & 0.00 & $0.24 \mathrm{~cd}$ & 0.14 & $0.72 \mathrm{~b}$ & 0.08 & $<0.01 \mathrm{~d}$ & 0.00 & $1.80 \mathrm{a}$ & 0.44 & $<0.01 \mathrm{~d}$ & 0.00 \\
\hline C22:1(n-9) & $<0.01 \mathrm{c}$ & 0.00 & $<0.01 \mathrm{c}$ & 0.00 & $<0.01 \mathrm{c}$ & 0.00 & $<0.01 \mathrm{c}$ & 0.00 & $<0.01 \mathrm{c}$ & 0.00 & $0.16 \mathrm{~b}$ & 0.13 & $0.92 \mathrm{a}$ & 0.14 \\
\hline C22:1(n-11) & $<0.01 \mathrm{~b}$ & 0.00 & $<0.01 \mathrm{~b}$ & 0.00 & $<0.01 \mathrm{~b}$ & 0.00 & $<0.01 \mathrm{~b}$ & 0.00 & $0.22 \mathrm{~b}$ & 0.13 & $0.17 \mathrm{~b}$ & 0.17 & $26.08 \mathrm{a}$ & 2.59 \\
\hline ¿MUFA & $14.13 \mathrm{f}$ & 1.51 & $18.77 \mathrm{e}$ & 5.02 & $26.94 \mathrm{~d}$ & 1.56 & $36.50 \mathrm{c}$ & 3.47 & $29.37 \mathrm{~d}$ & 1.80 & $45.40 \mathrm{~b}$ & 4.19 & $54.54 \mathrm{a}$ & 2.90 \\
\hline $\mathrm{C} 18: 2(\mathrm{n}-6)$ & $3.06 \mathrm{~cd}$ & 0.27 & $2.35 \mathrm{~d}$ & 0.99 & $3.34 \mathrm{c}$ & 0.62 & $6.41 \mathrm{~b}$ & 0.83 & $12.47 \mathrm{a}$ & 0.60 & $1.26 \mathrm{e}$ & 0.14 & $1.49 \mathrm{e}$ & 0.23 \\
\hline C18:3 $\gamma-\operatorname{lin}(n-6)$ & $0.39 \mathrm{~b}$ & 0.04 & $0.33 \mathrm{~b}$ & 0.06 & $0.24 \mathrm{c}$ & 0.04 & $0.49 a$ & 0.08 & $0.40 \mathrm{~b}$ & 0.08 & $0.17 \mathrm{~cd}$ & 0.04 & $0.11 \mathrm{~d}$ & 0.01 \\
\hline C20:2(n-6) & $0.83 \mathrm{a}$ & 0.10 & $0.27 \mathrm{c}$ & 0.04 & $0.82 \mathrm{a}$ & 0.19 & $0.77 \mathrm{a}$ & 0.09 & $0.50 \mathrm{~b}$ & 0.04 & $0.43 \mathrm{~b}$ & 0.08 & $0.20 c$ & 0.02 \\
\hline$C 20: 3(n-6)$ & $0.39 \mathrm{~b}$ & 0.09 & $0.36 \mathrm{~b}$ & 0.07 & $0.45 \mathrm{~b}$ & 0.11 & $1.01 \mathrm{a}$ & 0.10 & $0.44 \mathrm{~b}$ & 0.04 & $0.09 c$ & 0.03 & $<0.01 \mathrm{c}$ & 0.00 \\
\hline C20:4(n-6) & $9.47 \mathrm{a}$ & 0.64 & $7.67 \mathrm{~b}$ & 0.83 & $7.39 \mathrm{~b}$ & 1.92 & $5.53 \mathrm{c}$ & 0.68 & $0.97 \mathrm{e}$ & 0.10 & $2.82 \mathrm{~d}$ & 0.40 & $0.35 \mathrm{e}$ & 0.06 \\
\hline C22:5(n-6) & $1.33 \mathrm{~b}$ & 0.36 & $1.61 \mathrm{a}$ & 0.12 & $1.03 \mathrm{c}$ & 0.12 & $0.89 \mathrm{c}$ & 0.19 & $0.25 \mathrm{de}$ & 0.03 & $0.36 \mathrm{~d}$ & 0.06 & $0.10 \mathrm{e}$ & 0.03 \\
\hline C18:3(n-3) & $2.33 \mathrm{bc}$ & 0.56 & $1.79 \mathrm{~cd}$ & 0.44 & $2.74 \mathrm{~b}$ & 1.22 & $2.07 \mathrm{bc}$ & 0.51 & $7.67 \mathrm{a}$ & 0.17 & $0.63 \mathrm{e}$ & 0.11 & 1.15 de & 0.20 \\
\hline C18:4(n-3) & $0.12 \mathrm{c}$ & 0.05 & $0.26 \mathrm{c}$ & 0.05 & $0.27 \mathrm{c}$ & 0.27 & $0.27 \mathrm{c}$ & 0.12 & $1.39 \mathrm{~b}$ & 0.24 & $1.16 \mathrm{~b}$ & 0.21 & $2.87 \mathrm{a}$ & 0.56 \\
\hline $\mathrm{C} 20: 3(\mathrm{n}-3)$ & $0.94 \mathrm{a}$ & 0.16 & $0.31 \mathrm{~b}$ & 0.07 & $0.94 \mathrm{a}$ & 0.41 & $0.26 \mathrm{~b}$ & 0.05 & $0.30 \mathrm{~b}$ & 0.03 & $0.18 \mathrm{~b}$ & 0.04 & $0.09 \mathrm{~b}$ & 0.02 \\
\hline C20:4(n-3) & $0.72 \mathrm{ab}$ & 0.15 & $0.52 \mathrm{~b}$ & 0.11 & $0.92 \mathrm{a}$ & 0.68 & $0.63 \mathrm{ab}$ & 0.15 & $0.70 \mathrm{ab}$ & 0.09 & $0.35 \mathrm{~b}$ & 0.05 & $0.51 \mathrm{~b}$ & 0.08 \\
\hline C20:5(n-3) EPA & $10.51 \mathrm{~b}$ & 1.83 & $6.39 \mathrm{~cd}$ & 0.52 & $7.53 \mathrm{c}$ & 0.70 & $6.19 \mathrm{~cd}$ & 1.17 & $5.00 \mathrm{~d}$ & 0.44 & $12.58 \mathrm{a}$ & 1.55 & $5.40 \mathrm{~d}$ & 0.63 \\
\hline C22:5(n-3) & $3.21 \mathrm{a}$ & 0.54 & $2.52 \mathrm{a}$ & 0.53 & $2.77 \mathrm{a}$ & 1.35 & $2.69 \mathrm{a}$ & 0.43 & $1.30 \mathrm{~b}$ & 0.11 & $2.48 \mathrm{a}$ & 0.35 & $0.66 \mathrm{~b}$ & 0.15 \\
\hline C22:6(n-3)DHA & $19.63 \mathrm{~b}$ & 1.50 & $26.82 \mathrm{a}$ & 1.51 & $16.79 \mathrm{c}$ & 2.02 & $7.80 \mathrm{e}$ & 1.17 & $12.97 \mathrm{~d}$ & 2.13 & $7.67 \mathrm{e}$ & 0.87 & $7.61 \mathrm{e}$ & 0.87 \\
\hline ¿PUFA & $52.93 \mathrm{a}$ & 2.63 & $51.21 \mathrm{a}$ & 2.92 & $45.20 \mathrm{~b}$ & 3.16 & $35.01 \mathrm{c}$ & 3.35 & $44.36 \mathrm{~b}$ & 2.02 & $30.18 \mathrm{~d}$ & 2.92 & $20.54 \mathrm{e}$ & 1.27 \\
\hline$n-3 / n-6$ & $2.43 \mathrm{~cd}$ & 0.22 & $3.07 \mathrm{c}$ & 0.12 & $2.56 \mathrm{~cd}$ & 0.84 & $1.32 \mathrm{e}$ & 0.13 & $1.96 \mathrm{~d}$ & 0.22 & $4.93 \mathrm{~b}$ & 0.59 & $8.15 \mathrm{a}$ & 0.73 \\
\hline$\Sigma$ n-6 PUFA & $15.48 \mathrm{a}$ & 0.95 & $12.60 \mathrm{~b}$ & 0.91 & $13.26 \mathrm{~b}$ & 2.56 & $15.10 \mathrm{a}$ & 0.84 & $15.03 \mathrm{a}$ & 0.62 & $5.13 c$ & 0.63 & $2.26 \mathrm{~d}$ & 0.23 \\
\hline इn-3 PUFA & $37.46 \mathrm{a}$ & 2.45 & $38.62 \mathrm{a}$ & 2.10 & $31.94 \mathrm{~b}$ & 4.83 & $19.91 \mathrm{~d}$ & 2.66 & $29.33 \mathrm{~b}$ & 2.43 & $25.05 \mathrm{c}$ & 2.66 & $18.28 \mathrm{~d}$ & 1.16 \\
\hline En-3 HUFA & $35.00 \mathrm{a}$ & 2.27 & $36.57 \mathrm{a}$ & 2.55 & $28.94 \mathrm{~b}$ & 3.90 & $17.57 \mathrm{~d}$ & 2.60 & $20.26 \mathrm{~cd}$ & 2.55 & $23.26 \mathrm{c}$ & 2.56 & $14.27 \mathrm{e}$ & 0.86 \\
\hline OFA & $22.21 \mathrm{ab} a b$ & 1.52 & $23.01 \mathrm{a}$ & 1.91 & $21.33 \mathrm{ab}$ & 1.83 & $21.07 \mathrm{ab}$ & 1.18 & $18.26 \mathrm{c}$ & 0.62 & $20.34 b c$ & 1.19 & $22.92 \mathrm{a}$ & 2.56 \\
\hline DFA & $74.88 \mathrm{c}$ & 1.80 & $75.73 \mathrm{bc}$ & 1.93 & $77.33 \mathrm{ab}$ & 1.61 & $77.55 \mathrm{ab}$ & 1.18 & $73.91 \mathrm{c}$ & 0.66 & $78.46 \mathrm{a}$ & 1.25 & $76.07 \mathrm{bc}$ & 2.60 \\
\hline AI & $0.37 b c$ & 0.04 & $0.37 b c$ & 0.04 & $0.36 \mathrm{bc}$ & 0.02 & $0.36 \mathrm{bc}$ & 0.03 & $0.33 c$ & 0.01 & $0.41 \mathrm{~b}$ & 0.03 & $0.70 \mathrm{a}$ & 0.10 \\
\hline TI & $0.23 b c$ & 0.03 & $0.21 \mathrm{c}$ & 0.01 & $0.22 \mathrm{bc}$ & 0.05 & $0.31 \mathrm{a}$ & 0.03 & $0.16 \mathrm{~d}$ & 0.01 & $0.22 c$ & 0.02 & $0.26 \mathrm{~b}$ & 0.04 \\
\hline FLQ & 30.14 & 1.82 & 33.22 & 1.84 & 24.32 & 2.47 & 13.99 & 2.15 & 17.97 & 2.46 & 20.25 & 2.30 & 13.01 & 0.77 \\
\hline
\end{tabular}

$n$-Number of fish; SD—standard deviation; a, b, c, d, e, f-significant differences ( $p \leq 0.05$ ). The same letter (in rows) indicates the absence of significant differences ( $p>0.05$ ). $\Sigma$ SFA (saturated fatty acid), $\Sigma$ MUFA (monounsaturated fatty acid). The same letter (in rows) indicates the absence of significant differences $\mathrm{h}(p>0.05)$. EPA-eicosapentaenoic acid (C20:5).

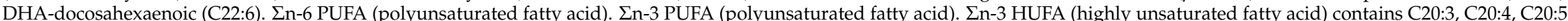
EPA, C22:5 and C22:6 DHA. AI-index of atherogenicity, TI—index of thrombogenicity, FLQ—flesh-lipid quality, OFA—hypocholesterolemic fatty acids, DFA—hypercholesterolemic fatty acids: "<" indicates that the contents are less than $0.01 \%$ 
Muscle tissue of perch and ide contained more mercury $(0.139$ and $0.109 \mathrm{mg} / \mathrm{kg}$, respectively) than other fish studied $(p \leq 0.05)$ (Table 1$)$, while a representative marine fish such as flounder had more mercury than herring $(0.021 \mathrm{mg} / \mathrm{kg})$, bream $(0.016 \mathrm{mg} / \mathrm{kg})$, rainbow trout $(0.015 \mathrm{mg} / \mathrm{kg})$ and carp $(0.006 \mathrm{mg} / \mathrm{kg})(p \leq 0.05)$. The differences in the content of mercury in muscles of herring, bream and rainbow trout were not significant $(p>0.05)$. However, the muscles of carp contained a significantly lower mercury concentration than other fish examined $(p \leq 0.05)$. The mercury content in muscles of the examined fish did not exceed maximum residue level $(0.5 \mathrm{mg} / \mathrm{kg})$.

Positive correlation coefficients were found between mercury levels in the muscles and the fish weight and length (Table 1). However, significant correlations were found between body weight and the content of mercury in muscle tissue of carp $(\mathrm{r}=0.878, p=0.050)$, flounder $(\mathrm{r}=0.925, p=0.008)$ and herring $(\mathrm{r}=0.982, p=0.0005)$.

Negative correlations were noted between the total length and the content of C18:2 (n-6) in muscles of bream $(\mathrm{r}=-0.845, p=0.034)$, as well as between the total length and AI $(\mathrm{r}=-0.890$, $p=0.018)$ or TI $(\mathrm{r}=-0.812, p=0.050)$ in muscles of ide (Table 3). The correlation coefficients between length and $\Sigma$ PUFA $(\mathrm{r}=0.835, p=0.038), \Sigma \mathrm{n}-3$ PUFA $(\mathrm{r}=0.821, p=0.045), \Sigma \mathrm{n}-3$ HUFA $(\mathrm{r}=0.836$, $p=0.038)$ or DFA $(r=0.837, p=0.038)$ in muscles of ide were significantly positive. There was also a positive correlation between length and C18:2(n-6) $(\mathrm{r}=0.927, p=0.024)$ in muscles of perch and the ratio $n-3 / n-6$ in muscle tissue of bream $(r=0.876, p=0.022)$. The content of $C 18: 2(n-6)$ in muscles of perch and carp was positively correlated with body weight $(\mathrm{r}=0.908, p=0.033$ and $\mathrm{r}=0.883, p=0.047$, respectively). Similarly, a positive correlation was observed between $\mathrm{C} 20: 5(\mathrm{n}-3)$ in bream $(\mathrm{r}=0.816$, $p=0.047)$, C14:0 in flounder $(\mathrm{r}=0.888, p=0.018)$ or $\Sigma$ MUFA in rainbow trout $(\mathrm{r}=0.835, p=0.039)$ and the body weight of these fish. However, there were negative correlation coefficients between body weight and C20:5(n-3), C20:5(n-3) in muscle tissue of perch of $\mathrm{r}=-0.930, p=0.022$ and $\mathrm{r}=-0.916$, $p=0.029$, respectively as well as $\Sigma$ PUFA, $\Sigma \mathrm{n}-3$ PUFA, $\Sigma \mathrm{n}-3$ HUFA and FLQ in muscles of rainbow trout $(\mathrm{r}=-0.887, p=0.018, \mathrm{r}=-0.845, p=0.034, \mathrm{r}=-0.831, p=0.040$ and $\mathrm{r}=-0.830, p=0.041$, respectively). 
Table 3. Linear correlation coefficients ( $r$ ) between fatty acids content in muscles of fish and body weight or total length.

\begin{tabular}{|c|c|c|c|c|c|c|c|c|c|c|c|c|c|c|c|c|}
\hline Fatty Acids & $\begin{array}{c}\text { Body } \\
\text { Weight } \\
\text { (r) }\end{array}$ & $p$ & $\begin{array}{c}\text { Total } \\
\text { Length } \\
\text { (r) }\end{array}$ & $p$ & $\begin{array}{c}\text { Body } \\
\text { Weight } \\
\text { (r) }\end{array}$ & $p$ & $\begin{array}{l}\text { Total } \\
\text { Length } \\
\text { (r) }\end{array}$ & $p$ & $\begin{array}{c}\text { Body } \\
\text { Weight } \\
\text { (r) }\end{array}$ & $p$ & $\begin{array}{l}\text { Total } \\
\text { Length } \\
\text { (r) }\end{array}$ & $p$ & $\begin{array}{c}\text { Body } \\
\text { Weight } \\
\text { (r) }\end{array}$ & $p$ & $\begin{array}{c}\text { Total } \\
\text { Length } \\
\text { (r) }\end{array}$ & $p$ \\
\hline Species & \multicolumn{4}{|c|}{ Bream } & \multicolumn{4}{|c|}{ Perch } & \multicolumn{4}{|c|}{ Ide } & \multicolumn{4}{|c|}{ Carp } \\
\hline C12:0 & -0.307 & ns & 0.005 & ns & 0.125 & ns & 0.173 & ns & -0.320 & $\mathrm{~ns}$ & -0.692 & $\mathrm{~ns}$ & 0.460 & ns & 0.557 & $\mathrm{~ns}$ \\
\hline C14:0 & -0.370 & ns & -0.071 & ns & -0.485 & ns & -0.407 & ns & 0.570 & ns & 0.292 & ns & 0.867 & ns & 0.544 & ns \\
\hline $\mathrm{C} 16: 0$ & -0.147 & ns & -0.092 & ns & -0.299 & ns & -0.136 & ns & -0.504 & ns & -0.672 & ns & 0.050 & ns & 0.029 & ns \\
\hline C18:0 & 0.153 & ns & 0.225 & ns & -0.783 & ns & -0.678 & ns & -0.200 & ns & -0.383 & ns & -0.239 & ns & 0.155 & $\mathrm{~ns}$ \\
\hline $\mathrm{C} 18: 2(\mathrm{n}-6)$ & -0.735 & ns & -0.845 & 0.034 & 0.908 & 0.033 & 0.927 & 0.024 & -0.182 & ns & -0.136 & ns & 0.883 & 0.047 & 0.603 & ns \\
\hline C20:4(n-6) & 0.361 & ns & 0.100 & ns & -0.806 & ns & -0.679 & ns & -0.743 & ns & -0.632 & ns & -0.551 & ns & 0.114 & $\mathrm{~ns}$ \\
\hline $\mathrm{C} 20: 3(\mathrm{n}-3)$ & 0.224 & ns & 0.439 & ns & 0.717 & ns & 0.708 & ns & 0.442 & ns & 0.521 & ns & 0.266 & ns & 0.414 & ns \\
\hline C20:4(n-3) & 0.112 & ns & 0.292 & ns & 0.523 & ns & 0.559 & ns & 0.510 & ns & 0.585 & ns & 0.498 & ns & 0.569 & ns \\
\hline $\mathrm{C} 20: 5(\mathrm{n}-3)$ & 0.816 & 0.047 & 0.724 & ns & -0.930 & 0.022 & -0.846 & ns & -0.189 & ns & 0.140 & ns & 0.020 & ns & 0.578 & ns \\
\hline $\mathrm{C} 22: 5(\mathrm{n}-3)$ & 0.722 & ns & 0.571 & ns & -0.916 & 0.029 & -0.876 & ns & 0.320 & ns & 0.621 & ns & -0.417 & ns & 0.265 & ns \\
\hline C22:6(n-3) & -0.216 & ns & -0.203 & ns & -0.254 & ns & -0.104 & ns & 0.124 & ns & 0.772 & ns & -0.336 & ns & 0.311 & ns \\
\hline$\Sigma$ SFA & -0.206 & ns & -0.088 & ns & -0.472 & ns & -0.313 & ns & -0.410 & ns & -0.759 & ns & 0.348 & ns & 0.450 & ns \\
\hline$\Sigma \mathrm{MUFA}$ & -0.598 & ns & -0.564 & ns & 0.363 & ns & 0.211 & ns & 0.557 & ns & -0.345 & ns & -0.198 & ns & -0.781 & ns \\
\hline इPUFA & 0.513 & ns & 0.396 & ns & -0.261 & ns & -0.122 & ns & 0.059 & ns & 0.835 & 0.038 & 0.087 & ns & 0.657 & ns \\
\hline$n-3 / n-6$ & 0.734 & ns & 0.876 & 0.022 & -0.687 & ns & -0.788 & ns & 0.563 & ns & 0.680 & ns & -0.229 & ns & 0.370 & ns \\
\hline$\Sigma$ n-6 PUFA & -0.322 & ns & -0.572 & ns & 0.064 & ns & 0.214 & ns & -0.685 & ns & -0.576 & ns & 0.359 & ns & 0.756 & ns \\
\hline OFA & -0.196 & ns & -0.100 & ns & -0.317 & ns & -0.157 & ns & -0.454 & ns & -0.756 & ns & 0.348 & ns & 0.224 & ns \\
\hline DFA & 0.289 & ns & 0.164 & ns & 0.351 & ns & 0.193 & ns & 0.457 & ns & 0.837 & 0.038 & -0.431 & ns & -0.371 & ns \\
\hline $\mathrm{AI}$ & -0.279 & ns & -0.114 & ns & -0.379 & ns & -0.226 & ns & -0.125 & ns & -0.890 & 0.018 & 0.707 & ns & 0.496 & ns \\
\hline TI & -0.375 & ns & -0.298 & ns & -0.417 & ns & -0.257 & ns & -0.458 & ns & -0.812 & 0.050 & 0.145 & ns & -0.301 & ns \\
\hline FLQ & 0.644 & ns & 0.562 & ns & -0.468 & ns & -0.322 & ns & 0.052 & ns & 0.687 & ns & -0.171 & ns & 0.482 & ns \\
\hline Species & \multicolumn{4}{|c|}{ Rainbow trout } & \multicolumn{4}{|c|}{ Flounder } & \multicolumn{4}{|c|}{ Herring } & & & & \\
\hline C12:0 & -0.280 & ns & -0.396 & ns & 0.247 & ns & -0.078 & ns & 0.088 & $\mathrm{~ns}$ & -0.335 & ns & & & & \\
\hline C14:0 & 0.735 & ns & 0.504 & ns & 0.888 & 0.018 & 0.632 & ns & -0.038 & ns & -0.212 & ns & & & & \\
\hline C16:0 & -0.170 & ns & -0.418 & ns & 0.109 & ns & -0.410 & ns & -0.258 & ns & -0.177 & ns & & & & \\
\hline C18:0 & 0.087 & ns & -0.122 & ns & 0.410 & ns & 0.015 & ns & 0.164 & ns & 0.280 & ns & & & & \\
\hline
\end{tabular}


Table 3. Cont.

\begin{tabular}{|c|c|c|c|c|c|c|c|c|c|c|c|c|c|c|c|c|}
\hline Fatty Acids & $\begin{array}{l}\text { Body } \\
\text { Weight } \\
\text { (r) }\end{array}$ & $p$ & $\begin{array}{l}\text { Total } \\
\text { Length } \\
\text { (r) }\end{array}$ & $p$ & $\begin{array}{l}\text { Body } \\
\text { Weight } \\
\text { (r) }\end{array}$ & $p$ & $\begin{array}{l}\text { Total } \\
\text { Length } \\
\text { (r) }\end{array}$ & $p$ & $\begin{array}{l}\text { Body } \\
\text { Weight } \\
\text { (r) }\end{array}$ & $p$ & $\begin{array}{l}\text { Total } \\
\text { Length } \\
\text { (r) }\end{array}$ & $p$ & $\begin{array}{c}\text { Body } \\
\text { Weight } \\
\text { (r) }\end{array}$ & $p$ & $\begin{array}{l}\text { Total } \\
\text { Length } \\
\text { (r) }\end{array}$ & $p$ \\
\hline C18:2(n-6) & 0.490 & ns & 0.533 & ns & 0.251 & ns & -0.166 & ns & -0.409 & ns & -0.056 & ns & & & & \\
\hline C20:4(n-6) & 0.119 & ns & -0.106 & ns & 0.406 & ns & 0.043 & ns & 0.118 & ns & 0.032 & ns & & & & \\
\hline C18:3(n-3) & -0.319 & ns & 0.024 & ns & -0.195 & ns & -0.142 & ns & -0.287 & ns & -0.190 & ns & & & & \\
\hline C20:3(n-3) & -0.135 & ns & -0.073 & ns & -0.155 & ns & -0.181 & ns & -0.379 & ns & -0.265 & ns & & & & \\
\hline C20:4(n-3) & 0.119 & ns & -0.106 & ns & -0.149 & ns & 0.348 & ns & -0.669 & ns & -0.447 & ns & & & & \\
\hline C20:5(n-3) & -0.805 & ns & -0.661 & ns & 0.120 & ns & 0.065 & ns & 0.343 & ns & 0.045 & ns & & & & \\
\hline C22:5(n-3) & -0.713 & ns & -0.437 & ns & -0.295 & ns & -0.031 & ns & 0.157 & ns & 0.095 & ns & & & & \\
\hline $\mathrm{C} 22: 6(\mathrm{n}-3)$ & -0.793 & ns & -0.719 & ns & 0.043 & ns & -0.350 & ns & -0.461 & ns & -0.309 & ns & & & & \\
\hline$\Sigma$ SFA & 0.436 & ns & 0.142 & ns & 0.357 & ns & -0.158 & ns & -0.156 & ns & -0.177 & ns & & & & \\
\hline$\Sigma$ MUFA & 0.835 & 0.039 & 0.753 & ns & -0.191 & ns & 0.105 & ns & 0.346 & ns & 0.341 & ns & & & & \\
\hline$\Sigma$ PUFA & -0.887 & 0.018 & -0.717 & ns & 0.076 & ns & -0.063 & ns & -0.465 & ns & -0.409 & ns & & & & \\
\hline$n-3 / n-6$ & -0.762 & ns & -0.697 & ns & -0.325 & ns & -0.016 & ns & 0.100 & ns & -0.341 & ns & & & & \\
\hline इn-6 PUFA & 0.425 & ns & 0.491 & ns & 0.339 & ns & -0.042 & ns & -0.448 & ns & -0.042 & ns & & & & \\
\hline$\Sigma$ n-3 PUFA & -0.845 & 0.034 & -0.721 & ns & 0.003 & ns & -0.060 & ns & -0.422 & ns & -0.440 & ns & & & & \\
\hline इn-3 HUFA & -0.831 & 0.040 & -0.740 & ns & 0.042 & ns & -0.079 & ns & -0.259 & ns & -0.311 & ns & & & & \\
\hline OFA & -0.037 & ns & -0.323 & ns & 0.307 & ns & -0.266 & ns & -0.171 & ns & -0.202 & ns & & & & \\
\hline DFA & -0.438 & ns & -0.144 & ns & -0.321 & ns & 0.210 & ns & 0.163 & ns & 0.188 & ns & & & & \\
\hline AI & 0.334 & ns & 0.003 & ns & 0.618 & ns & 0.031 & ns & -0.098 & ns & -0.192 & ns & & & & \\
\hline TI & 0.741 & ns & 0.468 & ns & 0.370 & ns & -0.152 & ns & -0.065 & ns & -0.057 & ns & & & & \\
\hline FLQ & -0.830 & 0.041 & -0.740 & ns & 0.097 & ns & -0.088 & ns & -0.242 & ns & -0.314 & ns & & & & \\
\hline
\end{tabular}

p-Significance levels for the correlation between the content of fatty acids in muscles of fish and their body weight or total length, ns-non-significant correlation. $\Sigma$ SFA (saturated fatty acid), $\Sigma$ MUFA (monounsaturated fatty acid), $\Sigma$ n-6 PUFA (polyunsaturated fatty acid), $\Sigma$ n-3 PUFA (polyunsaturated fatty acid), $\Sigma$ n-3 HUFA (highly unsaturated fatty acid) contains C20:3, C20:4, C20:5 EPA, C22:5 and C22:6 DHA. AI—index of atherogenicity, TI—index of thrombogenicity, FLQ—flesh-lipid quality, OFA—hypocholesterolemic fatty acids, DFA-hypercholesterolemic fatty acid. 


\section{Human Health Risk Assessment}

The THQ for mercury in different fish species is presented in Table 4. THQ values were below 1 which shows that there is no non-carcinogenic health risk to the consumer by consuming the examined fish. The EDI of mercury from the $33.16 \mathrm{~g}$ portions of fish was: $0.009 \mu \mathrm{g} /$ body weight (bream), 0.076 (perch), 0.060 (ide), 0.003 (carp), 0.008 (rainbow trout), 0.031 (flounder) and $0.012 \mu \mathrm{g} / \mathrm{body}$ weight (herring). The weekly intake of mercury (232.12 $\mathrm{g}$ of fish portion) accounts for 1.50, 13.34, 10.57, 0.60, $1.42,5.45,2.03 \%$ of the TWI (as $4 \mu \mathrm{g} / \mathrm{kg}$ body weight) and 4.611, 41.056, 32.527, 1.845, 4.363, 16.760 and $6.248 \%$ of the TWI (as $1.3 \mu \mathrm{g} / \mathrm{kg}$ body weight).

Table 4. The Hazard Quotient calculated for mercury content in the muscle tissue of fish.

\begin{tabular}{cccccc}
\hline Species & EDI & EWI & \%TWI * & \%TWI ** & THQ \\
\hline Bream (Abramis brama L.) $n=6$ & 0.0086 & 0.060 & 1.50 & 4.611 & 0.029 \\
Perch (Perca fluviatilis L.) $n=5$ & 0.0762 & 0.534 & 13.34 & 41.056 & 0.254 \\
Ide (Leuciscus idus L.) $n=6$ & 0.0604 & 0.423 & 10.57 & 32.527 & 0.201 \\
Carp (Cyprinus carpio L.) $n=5$ & 0.0043 & 0.024 & 0.60 & 1.845 & 0.011 \\
Rainbow trout & 0.0081 & 0.057 & 1.42 & 4.363 & 0.027 \\
(Oncorhynchus mykiss Walb.) $n=6$ & 0.0311 & 0.218 & 5.45 & 16.760 & 0.104 \\
Flounder (Platichthys flesus L.) $n=12$ & 0.0116 & 0.081 & 2.03 & 6.248 & 0.039 \\
Herring (Clupea harengus L.) $n=12$ &
\end{tabular}

$n$-Number of fish; EDI—the estimated daily intake ( $\mu \mathrm{g} / \mathrm{kg}$ body weight/day); EWI-the estimated weekly intake ( $\mu \mathrm{g} / \mathrm{kg}$ body weight/weekly); THQ-Target Hazard Quotient; * TWI-Tolerable Weekly Intake for inorganic mercury expressed as mercury $=4 \mu \mathrm{g} / \mathrm{kg}$ body weight, ${ }^{* *}$ TWI for methylmercury expressed as mercury is $1.3 \mu \mathrm{g} / \mathrm{kg}$ body weight.

\section{Discussion}

Previously findings reported by Łuczyńska and Krupowski [37] showed that mercury content in muscles of fish from the Polish market varied between some species. The muscles of predatory (perch and pike) and non-predatory freshwater fish (bream) contained higher levels of mercury than marine fish (flounder and mackerel) $(p \leq 0.05)$. Similarly, predatory freshwater fish (i.e., pike) had more mercury than non-predatory fish (i.e., bream). For bream and flounder, a contrary regularity was found (Table 1). Muscle tissue perch from the Vistula River (Torun, Poland) contained more mercury $(0.36 \mathrm{mg} / \mathrm{kg})$ than muscles of bream $(0.054 \mathrm{mg} / \mathrm{kg})$ [38]. Muscles tissue of flounder contained a higher concentration of mercury $(0.036 \mathrm{mg} / \mathrm{kg})$ than muscles of herring $(0.032 \mathrm{mg} / \mathrm{kg})$ [39]. Voigt [40] observed differences between the content of mercury in the muscles of the pelagic open-sea species (herring) and inshore species (perch) (Western Estonia). According to the same author perch contained more mercury than herring. The observations of above authors are close to those found in the present study. Kenšová et al. [41] also found the highest concentration of mercury in muscles of predatory fish (asp Aspius aspius L., eel Anguilla anguilla L., pike Esox lucius L., and perch). The same authors showed that among non-predatory fish (carp, bream, tench Tinca tinca L. and roach Rutilus rutilus L.) the lowest mercury content were noted in carp. Zrnčić et al. [42] studied 14 different fish species belonging to four groups according to feeding habits (among others: ide, carp and bream). The authors found that the differences between the content of metals examined, including mercury, in the four groups (herbavore, omnivore, piscivore and plankton-feeding fish) were significant. These results are consistent with those in present study (Table 1). Popov et al. reported that interspecies differences in the content of metals, including mercury, are most likely caused by peculiarities in the feeding habits of fish (Russia) [43]. According to those authors, the contents of mercury in muscles of perch, bream and ide were $0.033,0.035$ and $0.014 \mathrm{mg} / \mathrm{kg}$. There were statistically significant differences between content of mercury in muscles of herring $(0.0658 \mathrm{mg} / \mathrm{kg})$ and carp $(0.0373 \mathrm{mg} / \mathrm{kg})$ bought in Polish market $(p<0.05)$ [44]. Muscle tissue of carp from the Neretva River (Croatia) had higher values of mercury $(0.190 \mathrm{mg} / \mathrm{kg})$ than carp from the Polish market studied by the above authors [45]. The fish (carp and rainbow trout (Poland)) contained $0.036 \mathrm{mg} \mathrm{Hg} / \mathrm{kg}$ [46]. According to Mazej et al. [47] the muscles of 
perch from Velenjsko (Slovenia) had four times higher content than muscle tissue of carp $(0.03 \mathrm{mg} / \mathrm{kg})$. Lidwin-Każmierkiewicz et al. [48] found that muscle tissue of perch from West Pomerania (Poland) contained more mercury $(0.03 \mathrm{mg} / \mathrm{kg})$ than carp and bream $(0.01 \mathrm{mg} / \mathrm{kg})$. Those authors did not observe differences between the mercury concentration in muscles of carp and bream. However, Kenšová et al. [49] noted a significant differences between the content of mercury in muscles of carp and predatory fish species (asp Aspius aspius L., pike Esox Lucius L. and pikeperch Sander lucioperca L.) caught in the Vĕstonice Reservoir (Czech Republic) $(p<0.01)$, but did not find significant differences between the mercury concentration in muscle tissue of carp and bream $(p>0.01)$ or any dependence of metal content on fish weight, age or sex. For the muscle tissue of fish from Puck Buy, perch $(0.110-0.130 \mathrm{mg} / \mathrm{kg})$ contained higher values of mercury than other fish, i.e., bream $(0.040 \mathrm{mg} / \mathrm{kg})$ flounder $(0.031-0.053 \mathrm{mg} / \mathrm{kg})$ and herring $(0.049 \mathrm{mg} / \mathrm{kg})$ [50]. The same authors found a positive relationship $(p<0.05)$ only between total body length and weight and mercury content in muscles of flounder. Baeyens et al. [51] found strong positive correlation between the length of flounder and the concentration of mercury $(\mathrm{r}=0.71)$. This is in accordance with the results of the present study, but only in relation to correlation of mercury content in muscles of flounder with body weight.

The content of mercury in muscle of perch from Lake Gusinoye and the Selenga River (Russia) significantly depended on the fish length and weight $(\mathrm{r}=0.62-0.90, p<0.01)$ [52]. According to Łuczyńska [53], the positive correlation between the body weight and the total mercury levels in muscles of perch from Lake Łańskie, Pluszne, Dłużek and Maróz ( $\mathrm{r}=0.967,0.963,0.876$ and 0.967, $p<0.001$, respectively) was slightly higher than that between mercury and body length $(r=0.933$, $0.950,0.781$ and $0.916, p<0.001)$, respectively). A positive correlation between the concentration of mercury in muscles of perch from the southern Baltic and weight or length $(p<0.01)$ was found by Szefer et al. [54]. Mercury content in muscles of predatory fish belonging to five species (asp, Aspius aspius L.; eel Anquilla anquilla L.; perch; pike, Esox Lucius L.; pikeperch, Stizostedion lucioperca L.) from the Želivka Reservoir was correlated with weight $(r=0.330, p<0.001)$ [55]. The above findings were not confirmed by the results of this studyof perch.

There were also significant positive correlation between mercury content and fish body size (ide, carp and bream) [42]. A significant correlation coefficient between the concentration of mercury in muscle tissue of bream from Lake Balaton and their length $(r=0.8459, p<0.0001)$ was also observed by Farkas et al. [56]. This is in accordance with the examined results, but only in the case of carp.

According to Łuczyńska et al. [57], the content of fat and fatty acids varied both between and within species. The muscles of bream and perch contained 1.03 and 0.89 of total lipid, respectively. The fat content in fillets of carp and bream from Inland waters was $3.24 \%$ and $7.13 \%$, respectively [58]. These results are higher to those for the fish examined (Table 1). The muscle tissue of rainbow trout studied had lower content of fat than meat of rainbow trout from extensive farming $(3.13 \%)$ and intensive farming (5.39\%) [59] and fish of the same species from Polish market (6.84\%) [60]. Polak-Juszczak and Adamczyk [61] found that muscles of bream, perch and herring contained 3.14\%, $0.12 \%$ and $2.61 \%$ of fat. This literature data was not confirmed the presented findings.

Ljubojevic et al. [58] found differences between the content of MUFA, PUFA, n-3 PUFA and n-6 PUFA in muscles of bream and carp $(p<0.01)$. According to these authors fillets of bream contained more MUFA and n-3 PUFA than carp fillets, and lower amounts of PUFA and n-6 PUFA. These results are close to those for MUFA and n-3 PUFA in muscles of the fish studied (Table 2). Polak-Juszczak and Komar-Szymczak [62] studied the fatty acids profiles in muscles of bream, perch and herring from the Vistula Lagoon (Poland). They authors found that the content of SFA, MUFA and PUFA in muscle tissue of those fish were as follows: herring $>$ bream $\approx$ perch; bream $>$ herring $>$ perch and perch $>$ herring $>$ bream, respectively. In turn, Kołakowska et al. [63] observed that these groups in muscle lipids of rainbow trout, carp and flounder was as follows: flounder $>$ rainbow trout $>$ carp (SFA); carp $>$ flounder $>$ rainbow trout (MUFA) and rainbow trout $>$ flounder $>$ carp (PUFA). The results observed by Polak-Juszczak and Komar-Szymczak [62] and Kołakowska et al. [63] are in not accordance with the results of the present study. Similarly, the previously findings reported by Łuczyńska et al. [60] 
did not confirm the regularity of these fatty acids in muscles of carp, rainbow trout and bream from Polish market.

According to Ehsani et al. [64], monounsaturated fatty acids (MUFA) in fillets of rainbow trout were the highest, followed by polyunsaturated (PUFA) and saturated fatty acids (SFA). These results are not consistent with those of the present study, because the content of PUFA in muscles of the examined fish was higher than the content of MUFA (Table 2). Karaçali et al. [65] showed that MUFA in muscles of carp (Turkey), independent of seasonal variations, was at the higher amount $(45.67-50.17 \%)$ than SFA (25.29-28.13\%) and PUFA (17.87-26.73\%). These findings are not in agreement with those reported by Donmez [66] for muscles carp living in Porsuk Dam, Turkey (SFA > MUFA $\approx$ PUFA). However, Ćirković et al. [67] found that muscles of carp in raised in poly-culture (Serbia) had more PUFA than SFA and MUFA. According to these authors, nutrient composition, varies widely among fish species, especially the profile of fatty acids related to their consumption habits (herbivorous, omnivorous and carnivorous). Polak-Juszczak and Komar-Szymczak [62] observed that muscle tissue of bream had more MUFA than PUFA and SFA, whereas the muscle of herring had more PUFA than MUFA and SFA, and these groups in muscles of perch were as follows PUFA > SFA > MUFA. The results of authors are close only to those for perch examined. Stancheva et al. [68] noted that carp from the Danube River contained higher levels of n-3 PUFA in comparison with n-6 PUFA. These results are in good agreement with the data from the present study on the same fish species.

Ouraji et al. [69] and Stancheva et al. [68] reported that higher values of AI and TI (>1.0) are detrimental to human health. The value of these parameters in muscles of carp, both studied by Stancheva et al. [68] (0.65 and 0.36, respectively) and in the present study, were lower than 1.0. The FLQ value (6.84) in the muscle tissue of carp reported by Stancheva et al. [68] was lower than those noted in present study. Indices such as AI and TI in fillets of rainbow trout with three different average weights ranged from 0.20 to 0.28 and from 0.88 to 1.28 , respectively [64]. These values are superior to those for rainbow trout studied in the present study. The same author found that fish weighing about $480 \mathrm{~g}$ and $350 \mathrm{~g}$ contained lower AI and TI values than the low-weight fish, and that the content of DHA in fillets of rainbow trout decreased with weight. For the examined rainbow trout, DHA decreased with increased their weight, but the correlation was not significant.

A consumer who eats $232.12 \mathrm{~g}$ of weekly portion of fish meat ingests less mercury than the TWI [23], which means that it does not pose any health risks. The THQ of mercury in species examined for adult was also less than 1. Addo-Bediako et al. [70] reported that THQ $<1$ suggests that adverse health effects are unlikely, whereas THQ $>1$ suggests a high probability of adverse health effects. This shows that the fish from Polish market are safe for consumers. Although the greatest contribution of harmful exposure to mercury for humans is through eating fish, we should comprehensively address the subject and take into account the multiple sources of exposure. For example mercury content in fresh fruit and vegetables from independent agrarian production ranged between 0.0011 and $0.0039 \mathrm{mg} / \mathrm{kg}$ (Poland) [71]. However, vegetable products, products for infants and children and wheat cereal products contained $0.001-0.008 \mathrm{mg} / \mathrm{kg}$. These values did not affect health and were generally below the levels set forth in food legislation $(0.01 \mathrm{mg} / \mathrm{kg})$ [72]. The results observed by Duma et al. [73] also showed that the analysed products collected each year during 2002-2010 from selected farms in the Podkarpackie Province (Poland) did not present a risk to human health because the content of mercury $(<0.001-0.003 \mathrm{mg} / \mathrm{kg})$ in the tested products (milk, pigs, pork) did not exceed the maximum admissible values. Mercury, which has been acknowledged as a serious human toxin, was absent in the fruits and vegetable in Lagos state (Nigeria) [74]. Mercury content had no hazardous effect on human health and environment pollution in Shahre-Ray regions (Iran) because the mean concentrations of mercury in five leafy vegetables was $0.027 \mathrm{mg} / \mathrm{kg}$ dry weight [75]. The same authors found that mercury also had a low concentration in soil and water as compared with WHO/FDA references. The concentration of $\mathrm{Hg}$ in various vegetables (roots, stems, leafy, fruits, cereals and legumes) grown in four major industrial and urban cities (Tabouk, Riyadh, Damamm and Jazan) in Saudi Arabia was not detected in selected legume species from the northern district while it 
was at low levels in leafy vegetables (except parsley, which recorded the maximum mercury value $(0.048 \mathrm{mg} / \mathrm{kg}$ dry weight) [76]. According to these data, fish are more likely to be exposed to the toxic effects of mercury and contain more than other raw materials and food products.

\section{Conclusions}

The examined fish are better dietary sources of n-3 PUFA. Despite this, $n-3 / n-6$ ratio in marine fish was higher than other fish examined, which is associated with a small amount of $n-6$ PUFA in lipid muscles of this group. Furthermore, all fish species had more hypercholesterolemic fatty acids relative to hypocholesterolemic fatty acids and may be an important dietetic fish food from a cardiovascular disease point of view. The dietetic quality indices of lipids (index of flesh-lipid quality, atherogenicity and thrombogenicity) was no more than 1.0 which according to the data literature is detrimental to human health. In conclusion, the fish examined did not exceed the maximum acceptable level of mercury and were a beneficial source of PUFA, especially n-3 PUFA may be recommended for human health consumption, especially since the Target Hazard Quotient $(\mathrm{THQ}<1)$ showed there is a non-carcinogenic health risk to the consumer.

Acknowledgments: This study was financed by the Statutory Theme No. 17.610.008-300. Direct Mercury Analyzer Milestone's DMA-80 was financed by the project "Innovations in finfish aquaculture with special references to reproduction" (acronym: InnovaFish), Operational Programme Sustainable Development of the Fisheries Sector and Coastal Fishing Areas 2007-2013.

Author Contributions: Joanna Łuczyńska had the original idea for the study and wrote the manuscript, Beata Paszczyk and Joanna Łuczyńska collected and analyzed the data, Marek Jan Łuczyński and Joanna Łuczyńska conducted the statistical analysis, Beata Paszczyk contributed in the writing of the section "Analytical methods". Marek Jan Łuczyński contributed to the writing of the section "Results", Joanna Nowosad contributed to the manuscript revision process and prepared the description of other dietary $\mathrm{Hg}$ sources. All authors read and approved the final manuscript.

Conflicts of Interest: The authors declare no conflict of interest.

\section{References}

1. Belitz, H.-D.; Grosch, W.; Schieberle, P. Fish, whales, crustaceans, mollusks. Food Chem. 2009, 13, 617-639.

2. Breslow, J.L. n-3 Fatty acids and cardiovascular disease. Am. J. Clin. Nutr. 2006, 83, 1477-1482.

3. Williams, C.M. Dietary fatty acids and human health. Ann. Zootech. 2000, 49, 165-180. [CrossRef]

4. Kris-Etherton, P.M.; Harris, W.S.; Appel, L.J. Fish consumption, fish oil, omega-3 fatty acids, and cardiovascular disease. Circulation 2002, 2747-2757. [CrossRef]

5. Holub, D.J.; Holub, B.J. Omega-3 fatty acids from fish oils and cardiovascular disease. Mol. Cell. Biochem. 2004, 263, 217-225. [CrossRef] [PubMed]

6. Jańczyk, W.; Socha, P. Clinical implication of $\omega-3$ polyunsaturated fatty acids supplementation. Stand. Med. Pediatria 2009, 6, 10-17. (In Polish)

7. Raatz, S.K.; Silverstein, J.T.; Jahns, L.; Picklo, M.J. Issues of fish consumption for cardiovascular disease risk reduction. Nutrients 2013, 5, 1081-1097. [CrossRef] [PubMed]

8. Krzysik, M.; Biernat, J.; Grajeta, H. The influence of nutrients on immune system functioning-Part I. Immunomodulatory effects of fatty acids on the human body. Adv. Clin. Exp. Med. 2006, 15, 1055-1062.

9. Mensink, R.P.; Zock, P.L.; Kester, A.D.M.; Katan, M.B. Effects of dietary fatty acids and carbohydrates on the ratio of serum total to HDL cholesterol and on serum lipids and apolipoproteins: A meta-analysis of 60 controlled trials. Am. J. Clin. Nutr. 2003, 77, 1146-1155. [PubMed]

10. European Food Safety Authority (EFSA). Scientific opinion on dietary reference values for fats, including saturated fatty acids, polyunsaturated fatty acids, monounsaturated fatty acids, trans fatty acids, and cholesterol. EFSA J. 2010, 8, 1461. [CrossRef]

11. Yu, S.; Derr, J.; Etherton, T.D.; Kris-Etherton, P.M. Plasma cholesterol-predictive aquations demonstrate that stearic acid is neutral and monounsaturated fatty acids are hypocholesterolemic. Am. J. Clin. Nutr. 1995, 61, 1129-1139. [PubMed]

12. Nicolosi, R.J. Dietary fat saturation effects on low-density-lipoprotein concentrations and metabolism in various animal models. Am. J. Clin. Nutr. 1997, 65, 1617-1627. 
13. FAO Food and Nutrition Paper. Fats and Fatty Acids in Human Nutrition; Report of an Expert Consultation; Food and Agriculture Organization of the United Nations: Geneva, Switzerland, 2008.

14. Kris-Etherton, P.M.; Yu, S. Individual fatty acid effects on plasma lipids and lipoproteins: Human studies. Am. J. Clin. Nutr. 1997, 65, 1628-1644.

15. Helfrich, L.A.; Neves, R.J. Sustaining America's Aquatic Biodiversity. Freshwater Fish Biodiversity and Conservation. Available online: https:/ /vtechworks.lib.vt.edu/bitstream/handle/10919/54903/420-525. pdf? sequence $=1 \&$ is Allowed $=y$ (accessed on 25 May 2017).

16. Castro-González, M.I.; Méndez-Armenta, M. Heavy metals: Implications associated to fish consumption. Environ. Toxicol. Pharmacol. 2008, 26, 263-271. [CrossRef] [PubMed]

17. Da Silva Oliveira, A.L.; Barrocas, P.R.G.; de Counto Jacob, S.; Moreira, J.C. Dietary intake and health effects of selected toxic elements. Braz. J. Plant Physiol. 2005, 17, 79-93.

18. Díez, S. Human health effects of methylmercury exposure. In Reviews of Environmental Contamination Toxicology; Whitacre, D.M., Ed.; Springer: New York, NY, USA, 2008; Volume 198, pp. 111-132.

19. Park, J.-D.; Zheng, W. Human exposure and health effects of inorganic and elemental mercury. J. Prev. Med. Public Health 2012, 45, 344-352. [CrossRef] [PubMed]

20. Azevedo, B.F.; Furieri, L.B.; Peçanha, F.M.; Wiggers, G.A.; Vassallo, P.F.; Simões, M.R.; Fiorim, J.; de Batista, P.R.; Fioresi, M.; Rossoni, L.; et al. Toxic effects of mercury on the cardiovascular and central nervous systems. J. Biomed. Biotechnol. 2012, 1-11. [CrossRef] [PubMed]

21. Choi, A.L.; Grandjean, P. Methylmercury exposure and health effects in humans. Environ. Chem. 2008, 5, 112-120. [CrossRef]

22. Clarkson, T.W. Mercury: Major issues in environmental health. Environ. Health Persp. 1992, 100, 31-38. [CrossRef]

23. European Food Safety Authority (EFSA). Scientific Opinion on the risk for public health related to the presence of mercury and methylmercury in food. EFSA J. 2012, 10, 2985. [CrossRef]

24. Mahaffey, K.R. Fish and shellfish as dietary sources of methylmercury and the $\omega-3$ fatty acids, eicosahexaenoic acid and docosahexaenoic acid: Risks and benefits. Environ. Res. 2004, 95, 414-428. [CrossRef] [PubMed]

25. Domingo, J.L. Omega-3 fatty acids and the benefits of fish consumption: Is all that glitters gold? Environ. Int. 2007, 33, 993-998. [CrossRef] [PubMed]

26. Christie, W.W. (Ed.) The isolation of lipids from tissues. Recommended Procedures. Chloroform-methanol $(2: 1, \mathrm{v} / \mathrm{v})$ extraction and "Folch" wash. In Lipid Analysis. Isolation, Separation, Identification and Structural Analysis of Lipids; Pergamon Press Oxford: New York, NY, USA; Toronto, Japan; Sydney, Australia; Braunschweig, Germany, 1973; pp. 39-40.

27. Żegarska, Z.; Jaworski, J.; Borejszo, Z. Evaluation of the Peisker modified method for extracting methyl esters from fatty acids. Acta Acad. Agric. Techno. Olst. 1991, 24, 25-33. (In Polish)

28. Ulbricht, T.; Southgate, D. Coronary heart disease: Seven dietary factors. Lancet 1991, 338, 985-992. [CrossRef]

29. Garaffo, M.A.; Vassallo-Agius, R.; Nengas, Y.; Lembo, E.; Rando, R.; Maisano, R.; Dugo, G.; Giuffrida, D. Fatty acids profile, atherogenic (IA) and thrombogenic (IT) health lipid indices, of raw roe of blue fin tuna (Thunnus thunnus L.) and their salted product “Bottarga". Food Nutr. Sci. 2011, 2, 736-743. [CrossRef]

30. Telahigue, K.; Hajji, T.; Rabeh, I.; El Cafsi, M. The changes of fatty acid composition in sun dried, oven dried and frozen hake (Merluccius merluccius) and sardinella (Sardinella aurita). Afr. J. Biochem. Res. 2013, 7, 158-164. [CrossRef]

31. Abrami, G.; Natiello, F.; Bronzi, P.; McKenzie, D.; Bolis, L.; Agradi, E. A comparison of highly unsaturated fatty acid levels in wild and farmed eels (Anguilla anguilla). Comp. Biochem. Physiol. 1992, 101B, 79-81. [CrossRef]

32. Senso, L.; Suárez, M.D.; Ruiz-Cara, T.; Garcia-Gallego, M. On the possible effects of harvesting season and chilled storage on the fatty acid profile of the fillet of farmed gilthead sea bream (Sparus aurata). Food Chem. 2007, 101, 298-307. [CrossRef]

33. Statistical Yearbook of Agriculture. Food Economy, Consumption. Available online: stat.gov.pl/obszarytematyczne/roczniki-statystyczne/roczniki-statystyczne/rocznik-statystyczny-rolnictwa-2013,6,7.html (accessed on 27 February 2014). (In Polish)

34. Polak-Juszczak, L.; Nermer, T. Methylmercury and total mercury in eels, Anguilla anguilla, from Lakes in Northeastern Poland: Health risk assessment. EcoHealth 2016, 13, 582-590. [CrossRef] [PubMed] 
35. Ahmed, K.; Baki, M.A.; Kundu, G.K.; Islam, S.; Islam, M. Human health risks from heavy metals in fish of Buriganga river, Bangladesh. SpringerPlus 2016, 5, 1-12. [CrossRef] [PubMed]

36. US EPA. Regional Screening Levels (RSLs)—Generic Tables (June 2017). Available online: https: / / www.epa. gov / risk/regional-screening-levels-rsls-generic-tables-june-2017 (accessed on 28 July 2017).

37. Łuczyńska, J.; Krupowski, M. Mercury content in organs of commercial fish (Poland)—A short report. Pol. J. Food Nutr. Sci. 2009, 59, 345-348.

38. Wyrzykowska, B.; Falandysz, J.; Jarzyńska, G. Metals in edible fish from Vistula River and Dead Vistula River channel, Baltic Sea. J. Environ. Sci. Health Part B 2012, 47, 296-305. [CrossRef] [PubMed]

39. Pokorska, K.; Protasowicki, M.; Bernat, K.; Kucharczyk, M. Content of metals in flounder, Platichthys flesus L., and Baltic herring, Clupea harengus membras L., from the southern Baltic Sea. Arch. Pol. Fish. 2012, 20, 51-53. [CrossRef]

40. Voigt, H.-R. Heavy metal and organochlorine levels in coastal fishes from the Väike Väin Strait, western Estonia, in high summers of 1993-1994. Proc. Estonian Acad. Sci. Biol. Ecol. 2000, 49, 335-343.

41. Kenšová, R.; Kružiková, K.; Svobodová, Z. Mercury speciation and safety of fish from important fishing locations in the Czech Republic. Czech J. Food Sci. 2012, 30, 276-284.

42. Zrnčić, S.; Oraić, D.; Ćaleta, M.; Mihaljević, Ž.; Zanella, D.; Bilandžić, N. Biomonitoring of heavy metals in fish from the Danube River. Environ. Monit. Assess. 2013, 185, 1189-1198. [CrossRef] [PubMed]

43. Popov, P.A.; Vizer, A.M.; Androsova, N.V. Metal content in muscular tissue of commercially important fish from Novosibirsk Reservoir and near Dam on Ob’River. Contemp. Probl. Ecol. 2012, 4, 352-355. [CrossRef]

44. Szlinder-Richert, J.; Usydus, Z.; Malesa-Ciećwierz, M.; Polak-Juszczak, L. Marine and farmed fish on the Polish market: Comparison of the nutritive value and human exposure to PCDD/Fs and other contaminants. Chemosphere 2011, 85, 1725-1733. [CrossRef] [PubMed]

45. Has-Schön, E.; Bogut, I.; Strelec, I. Heavy metal profile in five fish species included in human diet, domiciled in the end flow of River Neretva (Croatia). Arch. Environ. Contam. Toxicol. 2006, 50, 545-551. [CrossRef] [PubMed]

46. Szkoda, J.; Nawrocka, A.; Kmiecik, M.; Żmudzki, J. Monitoring study of toxic elements in food of animal origin. Environ. Protect. Natur. Resour. 2011, 48, 475-484. (In Polish)

47. Mazej, Z.; Al Sayegh-Petkovšek, S.; Pokorny, B. Heavy metal concentrations in food chain of Lake Velenjsko, jezero, Slovenia: An artificial lake from mining. Arch. Environ. Contam. Toxicol. 2010, 58, 998-1007. [CrossRef] [PubMed]

48. Lidwin-Kaźmierkiewicz, M.; Pokorska, K.; Protasowicki, M.; Rajkowska, M.; Wechterowicz, Z. Content of selected essential and toxic metals in meat of freshwater fish from West Pomerania, Poland. Pol. J. Food Nutr. Sci. 2009, 59, 219-224.

49. Kenšová, R.; Čelechovská, O.; Doubravová, J.; Svobodová, Z. Concentrations of metals in tissues of fish from the Vĕstonice reservoir. Acta Vet. Brno. 2010, 79, 335-345. [CrossRef]

50. Boszke, L.; Siepak, J.; Falandysz, J. Total mercury contamination of selected organisms in Puck Bay, Baltic Sea, Poland. Pol. J. Environ. Stud. 2003, 12, 275-285.

51. Baeyens, W.; Leermakers, M.; Papina, T.; Saprykin, A.; Brion, N.; Noyen, J.; De Gieter, M.; Eiskens, M. Bioconcentration and biomagnification of mercury and methylmercury in North Sea and Scheldt estuary fish. Arch. Environ. Contam. Toxicol. 2003, 45, 498-508. [CrossRef] [PubMed]

52. Komov, V.T.; Pronin, N.M.; Mendsaikhan, B. Mercury content in muscles of fish of the Selenga River and Lakes of its basin (Russia). Inland Water Biol. 2014, 7, 178-184. [CrossRef]

53. Łuczyńska, J. The influence of weight and length on the mercury content in the muscle tissue of fish from four lakes in the Olsztyn Lake District (Poland). Arch. Pol. Fish. 2005, 13, 51-61.

54. Szefer, P.; Domagała-Wieloszewska, J.; Warzocha, J.; Garbacik-Wesołowska, A.; Ciesielski, T. Distribution and relationships of mercury, lead, cadmium, copper and zinc in perch (Perca fluviatilis) from the Pomeranian Bay and Szczecin Lagoon, southern Baltic. Food Chem. 2003, 81, 73-83. [CrossRef]

55. Kružiková, K.; Kenšová, R.; Sedláčková, L.; Jarkovskỳ, J.; Poleszczuk, G.; Svobodová, Z. The correlation between fish mercury liver/muscle ratio and high and low levels of mercury contamination in Czech localities. Int. J. Electrochem. Sci. 2013, 8, 45-56.

56. Farkas, A.; Salánki, J.; Specziár, A. Age- and size-specific patterns of heavy metals in the organs of freshwater fish Abramis brama L. populating a low-contaminated site. Water Res. 2003, 37, 959-964. [CrossRef]

57. Łuczyńska, J.; Borejszo, Z.; Łuczyński, M.J. The composition of fatty acids in muscles of six freshwater fish species from the Mazurian Great Lakes (northeastern Poland). Arch. Pol. Fish. 2008, 16, 167-178. [CrossRef] 
58. Ljubojevic, D.; Trbovic, D.; Lujic, J.; Bjelic-Cabrilo, O.; Kostic, D.; Novakov, N.; Cirkovic, M. Fatty acid composition of fishes from Inland waters. Bulg. J. Agric. Sci. 2013, 19, 62-71.

59. Skalecki, P.; Staszowska, A.; Kaliniak, A.; Florek, M. Utility value and meat quality of rainbow trouts (Oncorhynchus mykiss Walb.) from extensive and intensive farming. Rocz. Nauk. Pol. Tow. Zootech. 2013, 9, 59-67. (In Polish)

60. Łuczyńska, J.; Paszczyk, B.; Łuczyński, M.J. Fatty acid profiles in marine and freshwater fish from fish markets in northeastern Poland. Arch. Pol. Fish. 2014, 22, 181-188. [CrossRef]

61. Polak-Juszczak, L.; Adamczyk, M. Quality and amino acid composition of protein of fish from the Vistula Lagoon. Żywność Nauka Technol. Jakość 2009, 64, 75-83.

62. Polak-Juszczak, L.; Komar-Szymczak, K. Fatty acid profiles and fat contents of commercially important fish from Vistula Lagoon. Pol. J. Food Nutr. Sci. 2009, 59, 225-229.

63. Kołakowska, A.; Szczygielski, M.; Bienkiewicz, G.; Zienkowicz, L. Some of fish species as a source of $\mathrm{n} / 3$ polyunsaturated fatty acids. Acta Ichthyol. Piscat. 2000, 30, 59-70. [CrossRef]

64. Ehsani, A.; Jasour, M.S.; Khodayari, M. Differentation of common marketable-size rainbow trouts (Oncorhynchus mykiss) based on nutritional and dietetic traits: A comparative study. J. Appl. Anim. Res. 2013, 41, 387-391. [CrossRef]

65. Karaçali, M.; Bulut, S.; Konuk, M.; Solak, K. Seasonal variations in fatty acid composition of different tissues of mirror carp, Cyprinus carpio, in Orenler Dam Lake, Afyonkarahisar, Turkey. Int. J. Food Prop. 2011, 1007-1017. [CrossRef]

66. Donmez, M. Determination of fatty acid compositions and cholesterol levels of some freshwater fish living in Porsuk Dam, Turkey. Chem. Nat. Compd. 2009, 45, 14-17. [CrossRef]

67. Ćirković, M.; Ljubojević, D.; Đorđević, V.; Novakov, N.; Petronijević, R.; Matekalo-Sverak, V.; Trbović, D. The breed effect on productivity and meat nutrient composition of fish. Kafkas. Univ. Vet. Fak. Derg. 2012, 18, 775-780.

68. Stancheva, M.; Merdzhanova, A.; Dobreva, D.A.; Makedonski, L. Common carp (Cyprinus carpio) and European catfish (Silurus glanis) from Danube River as sources of fat soluble vitamins and fatty acids. Czech J. Food Sci. 2014, 32, 16-24.

69. Ouraji, H.; Shabanpur, B.; Abediankenari, A.; Shabani, A.; Nezami, A.; Sudagar, M.; Faghani, S. Total lipid, fatty acid composition and lipid oxidation of Indian white shrimp (Fenneropenaeus indicus) fed diets containing different lipid sources. J. Sci. Food Agric. 2009, 89, 993-997. [CrossRef]

70. Addo-Bediako, A.; Marr, S.M.; Jooste, A.; Luus-Powell, W.J. Human health risk assessment for silver catfish Schilbe intermedius Rüppell, 1832, from two impoundments in the Olifants River, Limpopo, South Africa. Water SA 2014, 40, 607-614. [CrossRef]

71. Bartodziejska, B.; Gajewska, M.; Czajkowska, A. Research on content of heavy metals contamination in independent agrarian production using atomic absorption spectrometry technique. Environ. Protect. Nat. Res. 2010, 43, 38-44. (In Polish)

72. Wojciechowska-Mazurek, M.; Starska, K.; Brulińska-Ostrowska, E.; Plewa, M.; Biernat, U.; Karłowski, K. Monitoring of contamination of foodstuffs with elements noxious to human health. Part 1 . Wheat cereal products, vegetable products, confectionery and products for infants and children (2004 year). Ann. Nation. Inst. Hyg. 2008, 59, 251-266. (In Polish)

73. Duma, P.; Pawlos, M.; Rudy, M. Contamination of selected food products by heavy metals In the Podkarpackie Province. Bromat. Chem. Toksykol. 2012, 45, 94-100.

74. Ogunkunle, A.T.J.; Bello, O.S.; Ojofeitimi, O.S. Determination of heavy metal contamination of street-vended fruits and vegetables in Lagos state, Nigeria. Int. Food Res. J. 2014, 21, 1725-1730.

75. Shirkhanloo, H.; Mirzahosseini, S.A.H.; Shirkhanloo, N.; Moussavi-Najarkola, S.A.; Farahani, H. The evaluation and determination of heavy metals pollution in edible vegetables, water and soil in the south of Tehran province by GIS. Arch. Environ. Prot. 2015, 41, 64-74. [CrossRef]

76. Ali, M.H.H.; Al-Qahtani, K.M. Assessment of some heavy metals in vegetables, cereals and fruits in Saudi Arabian markets. Egypt. J. Agric. Res. 2012, 38, 31-37. [CrossRef]

(C) 2017 by the authors. Licensee MDPI, Basel, Switzerland. This article is an open access article distributed under the terms and conditions of the Creative Commons Attribution (CC BY) license (http://creativecommons.org/licenses/by/4.0/). 